\title{
Discussion on Normalization Methods of Interval Weights
}

\author{
Yimeng Sui, Zhenyuan Wang \\ Department of Mathematics, University of Nebraska at Omaha, Omaha, United Stated \\ Email address: \\ ysui@unomaha.edu (Yimeng Sui), zhenyuanwang@unomaha.edu (Zhenyuan Wang)
}

\section{To cite this article:}

Yimeng Sui, Zhenyuan Wan. Discussion on Normalization Methods of Interval Weights. Science Journal of Applied Mathematics and Statistics. Vol. 4, No. 5, 2016, pp. 249-252. doi: 10.11648/j.sjams.20160405.19

Received: September 26, 2016; Accepted: October 12, 2016; Published: October 17, 2016

\begin{abstract}
This paper is collecting the classic and newly normalization methods, finding deficiency of existing normalization methods for interval weights, and introducing a new normalization methods for interval weights. When we normalize the interval weights, it is very important and necessary to check whether, after normalizing, the location of interval centers as well as the length of interval weights keep the same proportion as those of original interval weights. It is found that, in some newly normalization methods, they violate these goodness criteria. In current work, for interval weights, we propose a new normalization method that reserves both proportions of the distances from interval centers to the origin and of interval lengths, and also eliminates the redundancy from the original given interval weights. This new method can be widely applied in information fusion and decision making in environments with uncertainty.
\end{abstract}

Keywords: Normalization Methods, Weighted Average, Interval Weights, Information Fusion

\section{Introduction}

Multiple criteria decision analysis (MCDA) widespread in economics, management, engineering and military fields. MCDA is an important part of modern scientific decisionmaking and its theory and method have been widely used in engineering, economics, management and military as well as many other fields. The MCDA reasonably determined by the normalization of interval weight is very important because it is related to the reliability and accuracy of decision outcomes [2]. Therefore, the study of MCDA determined by the index weight has important theoretical and practical value. In the decision-making process, due to the complexity of the objective things, uncertainty and ambiguity of the human mind, to deal with a large number of uncertain data, the study under Multiple Attribute Decision Making with Uncertainty has important theoretical and practical significance. In the multi-attribute decision-making process to determine the attributes' weight is important research content [5]. Because of the uncertainty of attributes' weight, policymakers often use interval numbers or natural language to express the information on attributes' weight. As an aggregation tool in environment with uncertainty, the weighted average with interval weights is often used. Generally, to convert a weighted sum to be a weighted average, the given weights should be normalized. In literature, some normalization methods for interval weights have been proposed. However, there is not suitable criterion to judge the goodness of these normalization methods. Thus, proper normalized interval weights are useful and necessary in the field of science. The existing normalization methods are created by basic interval arithmetic. However, there is no criterion, which can be used to judge the goodness of these normalization methods, proposed in literature. Establishing a reasonable criterion and using it to judge normalization methods of interval weights is necessary. In this paper, checking an article written by Wang and Elhag [4], we discuss the goal and error of existing normalization methods. Furthermore, a criterion of reserving the proportion of interval weights unchanged is established. Then, we introducing a new good normalization method, which provides infinitely many reasonable normalized weights including two extreme weights: one with minimum uncertainty and another with maximum uncertainty.

After Introduction, this paper is organized as follows. In Section 2, we briefly recall some background and fundamental knowledge for interval weights. Then, the advantage to normalize the interval weights is discussed in Section 3. Also, we review some existing normalization for 
interval weights given in [4]. In Section 4, we point out the deficiency of existing normalization methods for interval weights. Through Section 5, a new methods to normalize interval weights is presented with a number of example. This paper is concluded with few comments in Section 6 .

\section{Preliminaries}

In this section, let us review some basic interval arithmetic law which would help and improve the further discussion. Let $a=\left[a^{-}, a^{+}\right]$and $b=\left[b^{-}, b^{+}\right]$be two non-negative intervals. By the extension principle, the following binary operations on the set of all non-negative intervals can be defined:

Addition: $a+b=\left[a^{-}+b^{-}, a^{+}+b^{+}\right]$;

Subtraction:

$$
a-b=\left[a^{-}-b^{+}, a^{+}-b^{-}\right] \text {if } a^{-}-b^{+} \geq 0 ;
$$

Multiplication: $a \times b=\left[a^{-} b^{-}, a^{+} b^{+}\right]$;

Division: $a \div b=\left[\frac{a^{-}}{b^{+}}, \frac{a^{+}}{b^{-}}\right]$if $b^{-} \neq 0$.

Also, we suppose that $w_{i}=\left[w_{i}^{-}, w_{i}^{+}\right]$for $i=1,2, \ldots, n$ are given interval weights and $\widehat{w}_{i}=\left[\widehat{w}_{i}{ }^{-}, \widehat{w}_{i}{ }^{+}\right]$for $i=$ $1,2, \ldots, n$, is called their normalized interval weights, where

$$
\begin{gathered}
\widehat{w}_{i}^{-}=\frac{w_{i}^{-}}{\sum_{j=1}^{n} w_{i}{ }^{+}}, i=1,2, \ldots, n, \\
{\widehat{w}_{i}}^{+}=\frac{w_{i}{ }^{+}}{\sum_{j=1}^{n} w_{i}}, i=1,2, \ldots,
\end{gathered}
$$

Once numerical information $\left\{x_{i} \mid i=1,2, \ldots, n\right\}$ for $n$ attributes is available, based on interval arithmetic, $\sum_{i=1}^{n} w_{i} x_{i}$ is the weighted sum. As a special case, if $\left\{w_{i} \mid i=1,2, \ldots, n\right\}$ is normalized, the weighted sum is called the weighted average (or, weighted mean).

In addition, Jimenez et al [3] suggested another normalization approach for interval weights, as follows:

$$
\begin{gathered}
k_{i}=\frac{w_{i}^{-}+w_{i}^{+}}{\sum_{j=1}^{n}\left(w_{i}^{-}+w_{i}^{+}\right)}, i=1,2, \ldots, n, \\
k_{i}^{-}=\frac{k_{i} w_{i}^{-}}{\frac{w_{i}^{-}+w_{i}^{+}}{2}}, i=1,2, \ldots, n, \\
k_{i}^{+}=\frac{k_{i} w_{i}^{+}}{\frac{w_{i}^{-}+w_{i}^{+}}{2}}, i=1,2, \ldots, n .
\end{gathered}
$$

where $k_{i}^{-}$and $k_{i}^{+}$form normalized weight interval $\left[k_{i}^{-}, k_{i}^{+}\right]$, $i=1,2, \ldots, n)$.

We can easily know the midpoints of interval weights through (3) to (5) as

$$
\begin{gathered}
k_{i}=\frac{\left(w_{i}^{-}+w_{i}^{+}\right)}{\sum_{j=1}^{n}\left(w_{i}^{-}+w_{i}^{+}\right)}, i=1,2, \ldots, n, \\
k_{i}^{-}=\frac{w_{i}^{-}}{\sum_{j=1}^{n}\left(w_{i}^{-}+w_{i}^{+}\right) / 2}, i=1,2, \ldots, n, \\
k_{i}^{+}=\frac{w_{i}^{+}}{\sum_{j=1}^{n}\left(w_{i}^{-}+w_{i}^{+}\right) / 2}, i=1,2, \ldots, n .
\end{gathered}
$$

\section{Newly Introduced Normalization Methods for Interval Weights}

Normalization can be divided into two categories: one is mapping the weights' value into interval $(0,1)$; another is transforming into dimensionless facilitate calculation and application. The latter uses some statistical probability distribution. According to the discussion in [4], interval weights $w_{i}=\left[w_{i}{ }^{-}, w_{i}{ }^{+}\right]$for $i=1,2, \ldots, n$ are normalized iff all $w_{i}^{-}$and $w_{i}{ }^{+}, i=1,2, \ldots, n$, are attainable. Here the meaning of "attainable" is that $w_{i}^{-}$(or $w_{i}^{+}$) can serve as $w_{i}$ in

$$
\begin{aligned}
N & =\left\{\left(w_{1}, w_{2}, \cdots, w_{n}\right) \mid w_{i}^{-} \leq w_{i}\right. \\
& \left.\leq w_{i}^{+}, i=1,2, \ldots, n, \sum_{i=1}^{n} w_{i}=1\right\}
\end{aligned}
$$

The purpose of such a definition is to remove redundancies. So, the authors of [4] want to use the maximum and minimum of interval weights vector as:

$$
\begin{aligned}
& \sum_{i=1}^{n} w_{i}^{-}+\max \left(w_{i}^{+}-w_{i}^{-}\right) \leq 1, \\
& \sum_{i=1}^{n} w_{i}^{+}+\max \left(w_{i}^{+}-w_{i}^{-}\right) \geq 1 .
\end{aligned}
$$

Moreover, the authors of [4] present normalization method for interval weights with the violation of condition. Let $\widehat{w}_{i}=\left[\widehat{w}_{i}{ }^{-}, \widehat{w}_{i}^{+}\right]$for $i=1,2, \ldots, n$.

$$
\begin{gathered}
\sum_{i=1}^{n} \widehat{w}_{i}^{-}+\max \left(\widehat{w}_{i}^{+}-\widehat{w}_{i}^{-}\right) \leq 1, \\
\sum_{i=1}^{n}{\widehat{w}_{i}}^{+}+\max \left(\widehat{w}_{i}^{+}-\widehat{w}_{i}^{-}\right) \geq 1 .
\end{gathered}
$$

If $\widehat{w}_{i}=\left[\widehat{w}_{i}^{-}, \widehat{w}_{i}^{+}\right]$for $i=1,2, \ldots, n$ are the interval weights determined by

$$
\begin{aligned}
& \widehat{w}_{i}^{-}=\frac{w_{i}^{-}}{w_{i}^{-}+\sum_{j \neq i}^{n} w_{i}^{+}}, i=1,2, \ldots, n, \\
& {\widehat{w}_{i}^{+}}^{+}=\frac{w_{i}^{+}}{w_{i}^{+}+\sum_{j \neq i}^{n} w_{i}^{-}}, i=1,2, \ldots, n,
\end{aligned}
$$

then

$$
\begin{gathered}
w_{i}^{-}=\max \left\{w_{i}^{-}, 1-\sum_{j \neq i} w_{j}^{+}\right\}, i=1,2, \ldots, n, \\
{w_{i}^{+}}^{+}=\min \left\{w_{i}^{+}, 1-\sum_{j \neq i} w_{j}^{-}\right\}, i=1,2, \ldots, n .
\end{gathered}
$$

Also, if an interval weight vector $W=\left(\omega_{1}, \ldots \ldots, \omega_{n}\right)$ with $\omega_{i}=\left[\omega_{i}{ }^{-}, \omega_{i}{ }^{+}\right]$and $\omega_{i}^{-} \leq \omega_{i}{ }^{+}$for $i=1,2, \ldots, n$ satisfies (15) and (16), let $\widehat{w}_{i}=\left[\widehat{w}_{i}{ }^{-}, \widehat{w}_{i}^{+}\right]$for $i=1,2, \ldots, n$, are the interval weights determined by

$$
\begin{gathered}
\widehat{w}_{i}^{-}=\max \left\{w_{i}^{-}, 1-\sum_{j \neq i} w_{j}^{+}\right\}, i=1,2, \ldots, n, \\
\widehat{w}_{i}^{+}=\min \left\{w_{i}^{+}, 1-\sum_{j \neq i} w_{j}^{-}\right\}, i=1,2, \ldots, n .
\end{gathered}
$$

Let $\widehat{w}_{i 1}=\left[\widehat{w}_{i 1}{ }^{-}, \widehat{w}_{i 1}{ }^{+}\right]$for $i=1,2, \ldots, n$, be the normalized interval weights determined by (17) and (18) and $\widehat{w}_{i 2}=\left[\widehat{w}_{i 2}{ }^{-}, \widehat{w}_{i 2}{ }^{+}\right]$be the normalized interval weights determined by (17) and (18). Then $\widehat{w}_{i 1}{ }^{-} \leq \widehat{w}_{i 2}{ }^{-}$and 
$\widehat{w}_{i 1}^{+} \geq \widehat{w}_{i 2}{ }^{+}$for $i=1,2, \ldots, n$.

\section{Deficiency of Existing Normalization Methods for Interval Weights}

Purpose in the article written by Wang and Elhag [4] is unclear. For normalizing interval weights, an additional criteria in necessary is to make the purpose clear reachable.

In paper [1] written by De-Qing $\mathrm{Li}$ et al, they cite a counterexample to the Lemma in paper [4]. In this Lemma, $\widehat{w}_{i}=\left[\widehat{w}_{i}{ }^{-}, \widehat{w}_{i}{ }^{+}\right]$for $i=1,2, \ldots, n$ are normalized interval weights determined by

$$
\begin{aligned}
& \widehat{w}_{i}^{-}=\max \left\{w_{i}^{-}, 1-\sum_{j \neq i} w_{j}^{+}\right\}, i=1,2, \ldots, n, \\
& \widehat{w}_{i}^{+}=\min \left\{w_{i}^{+}, 1-\sum_{j \neq i}{w_{j}}^{-}\right\}, i=1,2, \ldots, n .
\end{aligned}
$$

Then they conclude that there is only one interval $\widehat{w}_{k}=$ $\left[\widehat{w}_{k}{ }^{-}, \widehat{w}_{k}{ }^{+}\right], k \in\{1,2, \ldots, n\}$, which is different from the original interval $\widehat{w}_{k}=\left[\widehat{w}_{k}{ }^{-}, \widehat{w}_{k}{ }^{+}\right]$, while the others remain unchanged. Paper [1] gives a counterexample to disprove this conclusion. In the counterexample, three intervals $[0.4,0.6]$, $[0.3,0.6]$, and $[0.2,0.8]$ are used as the given weights. Then, according to the method shown in [4], the obtained normalized weights are $\widehat{w}_{1}=[0.4,0.5], \widehat{w}_{2}=[0.3,0.4]$ and $\widehat{w}_{3}=[0.2,0.3]$. In this example, all three intervals are changed. In our point of view, it also shows that this method cannot keep the proportion of the interval lengths unchanged, which is important in practice and we define it as a goodness criterion of normalization methods for interval weights in the next section, though the obtained new weights are reachable.

However, we can use a much simpler counterexample to negate the Lemma in [4] as follows.

Example 1. Consider only two attributes with interval weights $w_{1}=[0,2]$ and $w_{2}=[0,2]$. According to the method of normalization shown in [4], the normalized weights should be $\widehat{w}_{1}=\widehat{w}_{2}=[0,1]$. The original intervals are both changed. This negates the conclusion given in the Lemma of paper [4].

\section{Criterion of Goodness and a New Normalization Method for Interval Weights}

When we normalize the interval weights, it is very important and necessary to check whether the length of normalized interval weights keeps the same proportion with original intervals. It is found that, in papers [1] and [4], their methods violate such a goodness criterion. However, we can propose a new normalization method for interval weights that reserves the proportion of interval lengths and eliminate the redundancy for the original given intervals.

\subsection{Definition and Judgment of Normalization}

Let $w_{i}=\left[w_{i}^{-}, w_{i}^{+}\right], i=1,2, \ldots, n$, be interval weights with $0 \leq w_{i}^{-} \leq w_{i}^{+}$.

We assume

$$
N=\left\{\begin{array}{l|l}
\omega=\left(\omega_{1}, \omega_{2}, \ldots, \omega_{n}\right) & \begin{array}{c}
w_{i}^{-} \leq \omega_{i} \leq w_{i}^{+}, i=1,2, \\
\ldots, n ; \sum_{i=1}^{n} \omega_{i}=1
\end{array}
\end{array}\right\}
$$

to be the set of all normalized weight vectors. The following definition of normalization for interval weights can now be given.

Definition. [4] An interval weight vector $W=$ $\left(w_{1}, w_{2}, \ldots, w_{n}\right)$ with $w_{i}=\left[w_{i}^{-}, w_{i}^{+}\right]$and $0 \leq w_{i}^{-} \leq w_{i}^{+}$for $i=1,2, \ldots, n$, is said to be normalized if and only if it satisfies the following conditions:

(1) $N$ is nonempty;

(2) $w_{i}^{-}$and $w_{i}^{+}, i=1,2, \ldots, n$, are all attainable in $N$, that is, each of them can serve as an entry in some weight vector in $N$.

\subsection{Criterion of Goodness for Normalizing Interval Weights}

Let $w_{i}=\left[w_{i}{ }^{-}, w_{i}{ }^{+}\right]$for $i=1,2, \ldots, n$ be the given interval weights and $\widehat{w}_{i}=\left[\widehat{w}_{i}^{-}, \widehat{w}_{i}^{+}\right]$for $i=1,2, \ldots, n$ be its normalization. The latter should has the same proportion of interval lengths as the former, that is, $\widehat{w}_{i}^{+}-\widehat{w}_{i}{ }^{-}=$ $k\left(w_{i}{ }^{+}-w_{i}{ }^{-}\right)$for all $i=1,2, \ldots, n$, where $k$ is a nonnegative number.

Based on this goodness criterion, a new method of normalization for interval weights can now be introduced.

Three steps of new method to normalize interval weights:

(1) Finding the midpoint of each interval weight $w_{i}$, denoted by $m_{i}$, that is,

$$
m_{i}=\frac{w_{i}{ }^{+}-w_{i}^{-}}{2}, i=1,2, \ldots, n ;
$$

(2) Regarding $m_{i}, i=1,2, \ldots, n$, as real-valued weights, using classical method to normalize them, and denoting the obtained normalized real-valued weights by $\omega_{i}, i=1,2, \ldots, n$

(3) According to the proportion of the length of original interval weights, extending them to be intervals as large as possible but not violating the attainability described in [4].

The third step can be realized by solving an LP maximization problem with respect to parameter $t \geq 0$ as follows. Let $l_{i}=w_{i}{ }^{+}-w_{i}{ }^{-}, i=1,2, \ldots, n$. $\max t$

$$
\begin{gathered}
\text { s.t. } \omega_{i_{i}}+t l_{i}+\sum_{j \neq i}\left(\omega_{i_{j}}-t l_{j}\right) \leq 1, i=1,2, \ldots, n \\
\omega_{i_{i}}-t l_{i}+\sum_{j \neq i}\left(\omega_{i_{j}}+t l_{j}\right) \geq 1, i=1,2, \ldots, n \\
\omega_{i_{i}}+t l_{i} \leq 1, i=1,2, \ldots, n \\
\omega_{i}-t l_{i} \geq 0, i=1,2, \ldots, n
\end{gathered}
$$




$$
t \geq 0 \text {. }
$$

Denote the solution of above LP problem by $t_{m}$. Taking $t \in\left[0, t_{m}\right]$, any interval weights $\left[\omega_{i_{i}}-t l_{i}, \omega_{i}+t l_{i}\right]$, $i=1,2, \ldots, n$ is a normalization of given interval weights $w_{i}=\left[w_{i}{ }^{-}, w_{i}{ }^{+}\right]$for $i=1,2, \ldots, n$. When $t_{m}=0$, the normalization of interval weights $w_{i}=\left[w_{i}{ }^{-}, w_{i}{ }^{+}\right]$for $i=1,2, \ldots, n$ is unique. While $t_{m}>0$, there are infinitely many normalizations for the original interval weights. All of these normalizations satisfy the requirement in abovementioned goodness criterion. Weights $\omega_{i_{i}}, i=1,2, \ldots, n$, called the minimum normalization of the given interval weights, has the smallest uncertainty, that is, it is just the classical weights. Weights $\left[\omega_{i_{i}}-t_{m} l_{i}, \omega_{i_{i}}+t_{m} l_{i}\right], i=$ $1,2, \ldots, n$, called the maximum normalization of the given interval weights, has the largest uncertainty. Users can select any one from them according to the real situation of the application.

\subsection{Example to Testify the New Method}

Example 2. We still used the interval weights given in Example 1. By the new method, from interval weights $[0,2]$ and $[0,2]$ with $l_{1}=l_{2}=2$, we can get the minimum normalization 0.5 and 0.5 . The maximum normalization is [0, $1]$ and $[0,1]$ with $t=t_{m}=1$. It is easy to see that they are both attainable and reserve the proportion of the interval lengths. In the following examples, using an $n$-tupe to express a weights is convenient.

Example 3. Given interval weights $([0,2],[2,5],[5,9])$. The midpoints are $1,3.5,5$ respectively. The normalized interval weights should have expression $\left(\frac{2}{23} \pm t, \frac{7}{23} \pm t, \frac{14}{23} \pm\right.$ $t)$. Considering the restriction in the LP problem, the minimum normalization is $\left(\frac{2}{23}, \frac{7}{23}, \frac{14}{23}\right)$, while the maximum normalization is $\left(\left[0, \frac{4}{23}\right],\left[\frac{4}{23}, \frac{10}{23}\right],\left[\frac{10}{23}, \frac{18}{23}\right]\right)$ with $t=t_{m}=\frac{1}{23}$.

Example 4. Consider given interval weights $([0,1],[1,2]$, $[2,3],[3,4])$. According to above new method, we can find the midpoints being $0.5,1.5,2.5,3.5$. Any normalized weights can be expressed as $\left(\frac{1}{16} \pm t, \frac{3}{16} \pm t, \frac{5}{16} \pm t, \frac{7}{16} \pm t\right)$. Finally, through solving the LP problem, the maximum normalization is interval weights $\left(\left[0, \frac{1}{8}\right],\left[\frac{1}{8}, \frac{1}{4}\right],\left[\frac{1}{4}, \frac{3}{8}\right],\left[\frac{3}{8}, \frac{1}{2}\right]\right)$. It is notable that not any interval weights has infinitely many normalizations.

Example 5. Regarding any real number $a$ as an interval $[a$, $a]$, we take interval weights $(0.3,[0.1,0.3])$ with midpoints 0.3 , and 0.2 . Since the length of interval $[0.3,0.3]$ is 0 , the normalized interval weights should have an expression $(0.6,0.4 \pm t)$. In this case, $t_{m}=0$, that is, the unique normalization is classical weights $(0.6,0.4)$.

It is evident that this new method can also reserve the proportion of the distances from the centers of intervals to the origin. This is an intuitive goodness of the new method discussed in this section and should be regarded as a basic requirement for normalization of interval weights, ever for classical weights.

\section{Conclusion}

The normalization of interval weights is important and necessary in multiple criteria decision analysis (MCDA) problems. There already exist some normalization methods for interval weights. However, no criterion has been used to judge their goodness. In this work, we introduce a goodness criterion that is intuitive and practicable. Under this criterion, we can see that those existing normalization methods are not good. Then, we introduce a new method for normalizing interval weight. This new method is good enough in practice since it reserves the proportions for both locations of interval centers and lengths of intervals from the original interval weights, and it can delete all redundancy.

\section{References}

[1] D. -Q. Li, J. -Y. Wang, and H. -X. Li, Note on "The normalization of interval and fuzzy weights", Fuzzy Sets and Systems 160 (2009) 2722-2725.

[2] O. Pavlacka, On various approaches to normalization of interval and fuzzy weights, Fuzzy Sets and Systems 243 (2014) $110-130$.

[3] P. Sevastjanov, L. Dymova, and P. Bartosiewicz, A new approach to normalization of interval and fuzzy weights, Fuzzy Sets and Systems 198 (2012) 34-45.

[4] Y. -M. Wang, and T. M. S. Elhag, On the normalization of interval and fuzzy weights, Fuzzy Sets and Systems 157 (2006) 2456-2471.

[5] Z. Wang, R. Yang, and K. -S. Leung, Nonlinear integrals and their Applications in Data Mining, World Scientific, 2010. 